\title{
MAMPOSTERÍA DE BLOQUES DE SUELO CEMENTO INTER TRABADOS CONFINADA EN UNA ESTRUCTURA DE HORMIGÓN ARMADO
}

\section{MASONRY INTERLOCKING CONCRETE EARTH BLOCKS (CEB) CONFINED IN A REINFORCED CONCRETE STRUCTURE}

Dol: https://doi.org/10.47796/ra.2020i17.356

EDUARDO ENRIQUE BRIZUELA ${ }^{1}$
PRESENTAD0 : 06.05 .20

ACEPTADO : 01.07 .20

\section{RESUMEN}

A partir de la adquisición de una prensa para bloques de suelo que se encontraba en desuso y en el marco del proyecto de un taller para una pequeña empresa constructora, se realiza esta investigación sobre selección de suelos y estabilizantes apropiados para la producción de bloques de suelo cemento; la ejecución de mamposterías con estos bloques y el estudio de costos comparados con otras mamposterías tradiciones.

PALABRAS CLAVE: Suelo cemento, bloques, mamposterías, producción.

\section{ABSTRACT}

From the acquisition of a press for Concrete Earth Blocks (CEB) that is in disuse and as part of the project of a workshop for a small construction company, this research is carried out on the production process of these Interlocked Concrete Earth Blocks, the selection of suitable soils and stabilizers selected for its elaboration, the execution of masonry with this type of block, the study of its production costs and comparison with the costs of other masonry.

KEYWORDS: Cement floor, blocks, masonry, production.

Arquitecto, director del Laboratorio de construcciones con Tierra Cruda, profesor a cargo de la materia Construcciones con Tierra Cruda, profesor adjunto de la material Taller de Diseño Arquitectónico II, Escuela de Arquitectura, Universidad Nacional de La Rioja, profesional independiente, miembro de la red PROTIERRAArgentina. 


\section{INTRODUCCIÓN}

Según el Instituto Mexicano del Cemento y el Concreto:

La aplicación de suelo cemento ${ }^{2}$ empezó a estudiarse metódicamente entre 1910 y 1920. En Inglaterra, en 1917, Brooke Bradley empleó con éxito una mezcla de cemento con suelos arcillosos en la construcción de carreteras. Sin embargo, a pesar de los excelentes resultados, la técnica no fue usada posteriormente. En los Estados Unidos, el uso del suelo - cemento se incrementó a partir de la patente de Joseph Hay Amies en 1917, de una mezcla de suelo con cemento llamada Soilamiesha siendo utilizado en obra viales y en diques en los Estados Unidos de América desde 1930 en los Estados Unidos, su empleo experimental en la construcción de Viviendas es posterior. ( $p 1$ )

La tecnología del BTC $^{3}$ fue desarrollada durante la década de 1950 por el Centro Interamericano de Vivienda y Planeamiento de Colombia y es el resultado de una investigación que tenía por objetivo producir materiales de construcción de bajo costo. Nace así la primera prensa de suelo, la CINVA-RAM, nombrada así en honor a su inventor, el Ing. Raúl Ramírez. Desde la década de 1980 la tecnología del bloque de tierra comprimida ha tenido gran difusión en todo el mundo, en especial en los países en vías de desarrollo.

El estudio sobre del $\mathrm{BSC}^{4}$ adquiere relevancia a partir de la aparición en el medio local de emprendimiento dedicados a la producción y comercialización de este material, también denominados bloques ecológicos y a su difusión como una alternativa de construcción sostenible.

El objetivo de este artículo es exponer las potencialidades de este sistema constructivo de tierra, el cual es una alternativa a las mamposterías tradicionales por su bajo costo, impacto ambiental y empleo de recursos locales.

La metodología empleada en esta investigación es cuanti/cualitativa, entendiéndose por cuantitativos a los procedimientos y análisis estadísticos realizados para establecer los suelos y estabilizantes apropiados para la elaboración de los bloques; por cualitativos a los procedimientos subjetivos realizados para mejorar la producción de la prensa y ejecutar la mampostería, con datos que se recogen a lo largo de un espacio de tiempo, combinando ambos métodos.

\section{MATERIALES Y MÉTODOS}

Esta investigación utiliza el método empírico, realizándose en una primera instancia una recopilación documental sobre esta técnica constructiva y la maquinaria necesaria para su ejecución. Así también se indagó sobre los suelos y estabilizantes apropiados para su producción, realizando pruebas de campo. A través de la observación directa se seleccionó las trabas y el mortero de asiento adecuados para la ejecución de la mampostería.

\section{DESARROLLO}

\section{Reacondicionamiento de la Prensa}

La prensa utilizada funciona mediante un sistema mecánico de palancas de accionamiento manual fabricada por la firma

\footnotetext{
El Suelo cemento o suelo estabilizado con cemento es una mezcla en seco de suelo o tierra con determinadas características granulométricas, cemento Portland y en algunos casos aditivos. A la mezcla se le adiciona una cierta cantidad de agua para su fraguado y posteriormente se compacta.

3 BTC, Bloque de tierra comprimida, (CEB en Ingles) es un material de construcción fabricado con una mezcla de tierra y un material estabilizante, como cal aérea, cal hidráulica, cemento 0 arcilla, que es comprimida y moldeada utilizando una prensa.

BSC Bloque de Suelo Cemento, denominación que se utiliza para distinguir al bloque de tierra comprimida solo estabilizado con cemento.
} 
metalúrgica Ricardo Marchese. Produce bloques de suelo comprimidos inter trabados de $28 \times 14 \times 9 \mathrm{~cm}$ de alto, machihembrado tanto en la junta vertical como horizontal, conocidos comercialmente como bloques Ladritrac.

Al ser adquirida presentaba diferentes problemas causados por defectos de fabricación y desgastes de sus partes móviles producto del uso, las cuales fueron reemplazadas. No estaba sujeta a base alguna, lo cual la hacía inestable y de difícil traslado, situación resuelta fijándola a una base metálica con manijas y ruedas. (Fig. 1)

Fue montada sobre la prensa una tolva y cajón para cargar para el material, mejorando asíla producción diaria de la misma. (Fig. 2)

\section{Figura 1. Fijación a una base metálica para transporte}

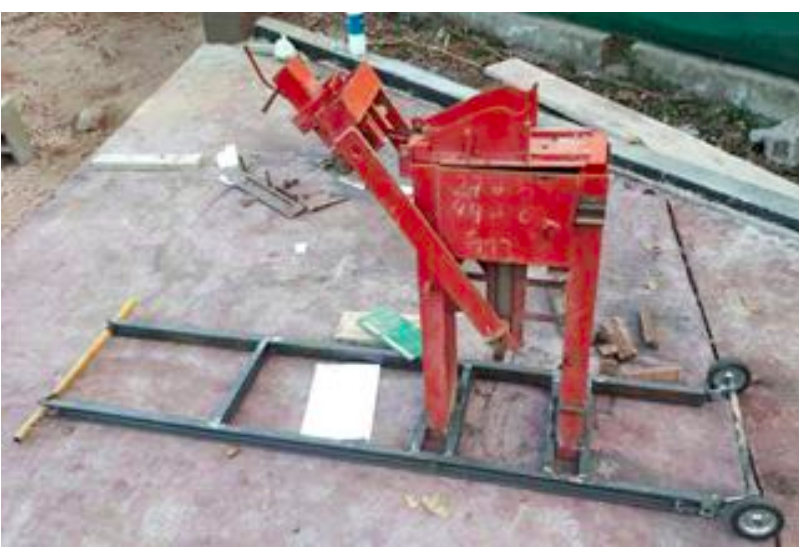

Fuente: Fotografía del autor

Selección de suelos para la producción de los bloques

Para seleccionar los suelos a utiliza se realizaron las pruebas de campo indicadas en el documento de la Red Proterra "Selección de Suelos y Métodos de Control en la Construcción con Tierra, prácticas de campo". En las mencionadas pruebas se determinó un alto
Figura 2. Agregado de tolva y cajón de carga

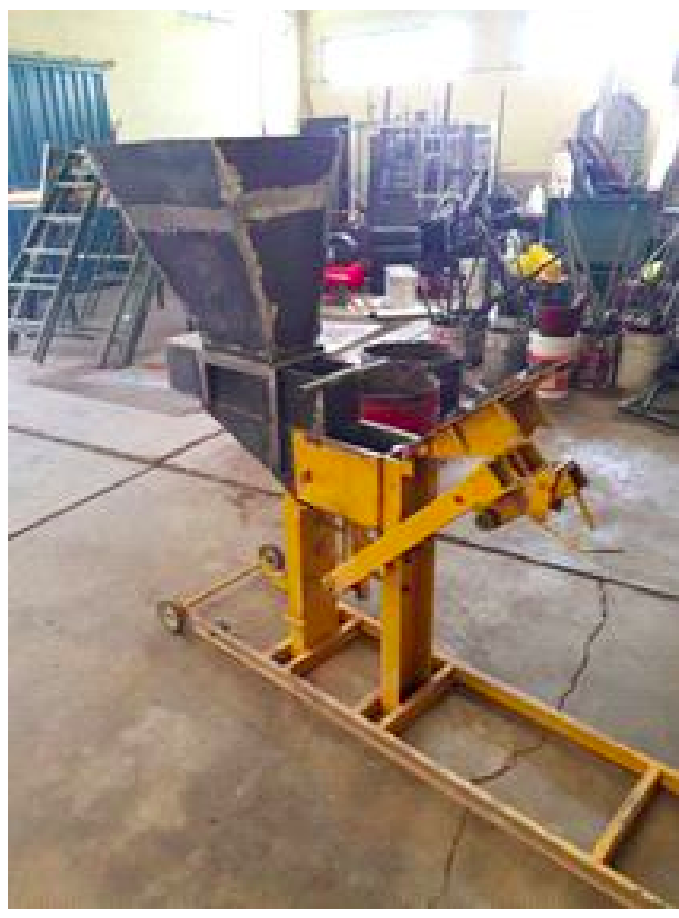

Fuente: Fotografía del autor.

contenido de limos y baja presencia de arcillas en el suelo del lugar, por lo que se optó por utilizar el suelo del cauce de un río cercano compuesto por gravas, arenas gruesas y finas, el cual fue pasado por un tamiz de malla, para eliminar el la grava.

A las arenas gruesas y finas obtenidas de este proceso se le agregó arcilla de una cantera distante $50 \mathrm{~km}$ del lugar para lograr la cohesión de las partículas de arena durante el moldeo inicial, lo que permite retirar el bloque de la prensa y estibarlo de manera ágil y segura.

\section{Selección de Estabilizantes}

Se realizaron bloques de pruebas con mezclas del suelo seleccionado y distintos estabilizantes como la cal hidratada (hidróxido de calcio), el cemento portland ${ }^{5}$ y cemento de albañilería ${ }^{6}$. (Fig. 3)

\footnotetext{
Conglomerante hidráulico, que contiene al clínker pórtland como constituyente necesario, pequeñas cantidades de sulfato de calcio y la eventual adición de hasta un $10 \%$ de escoria granulada de alto horno. Es un material inorgánico que, una vez endurecido, conserva su resistencia y estabilidad incluso bajo el agua

6 El Cemento de Albañilería es un producto que se obtiene de la molienda conjunta de clinker portland, adiciones minerales y aditivos que mejoran la plasticidad, trabajabilidad, adherencia y retención de agua, convirtiéndolo en un producto apto para obras de albañilería, reemplazando a los morteros tradicionales de Cemento y Cal
} 
Figura 3. Serie de bloques de prueba

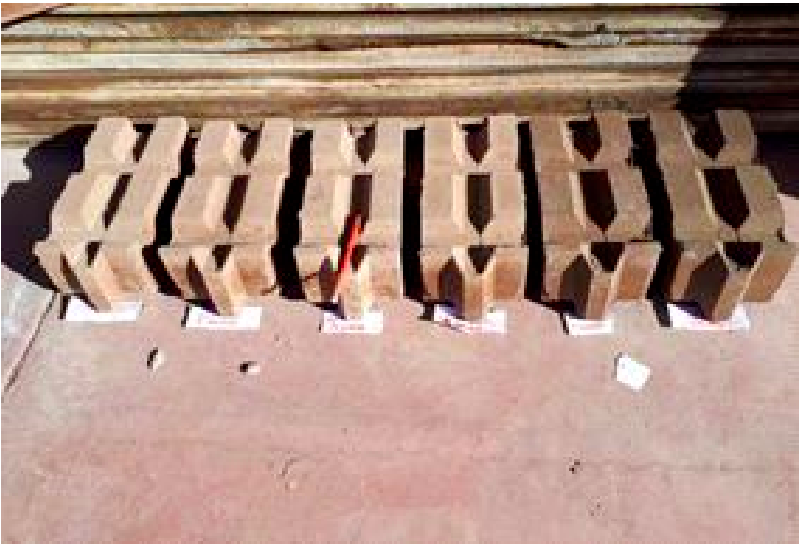

Fuente: Fotografía del autor.

En la primera mezcla el suelo seleccionado fue pasado por un tamiz de maya de $5 \mathrm{~mm}$. de apertura, el cual no eliminó en su totalidad las gravas, observándose a simple vista un problema de desgranamiento en las aristas del bloque de prueba.

La segunda mezcla se realizó con dos partes de suelo pasado por tamiz $5 \mathrm{~mm}$. y una parte de suelo pasada por tamiz de $2 \mathrm{~mm}$. de apertura y estabilizado con cal hidratada al $10 \%$ y cemento al \%10, observando un menor desgranamiento del bloque de prueba.

En la tercera mezcla se utilizó un suelo con las mismas características de la mezcla 2, pero la cal hidratada fue reemplazada por cemento de albañilería en la misma proporción, persistiendo el problema del desgranamiento de los bordes pero logrando una mayor resistencia.

En la cuarta mezcla todo el suelo fue pasado por el tamiz de maya de $2 \mathrm{~mm}$, eliminándose por competo las gravas, volviéndose a utilizar con cal hidratada y cemento como estabilizante. Durante el proceso de secado surgiendo fisuras en toda su superficie del bloque, deduciéndose que la cal hidratada no alcanzó a neutralizar los efectos retroactivos de la arcilla agregada al suelo.

La quinta mezcla fue hecha con suelo pasado por el tamiz de malla de $2 \mathrm{~mm}$. de apertura, cemento de albañilería al $15 \%$ y cemento portland al 5\%, siendo el bloque elaborado con esta mezcla, el que alcanzo la mayor resistencia mecánica.

En la sexta mezcla se disminuyó la cantidad de arcilla agregada para despejar dudas si las fisuras detectadas en la cuarta mezcla fueron producidas por un elevado contenido de arcilla en el suelo o por la baja calidad de la cal hidratada empleada para estabilizarlo. El bloque elaborado con esta mezcla no presento fisuras pero si poca manejabilidad al ser extraído de la prensa. (Tabla 1)

Tabla 1. Mezclas de suelo y estabilizantes empleados en proporciones

\begin{tabular}{|c|c|c|c|c|c|c|c|c|c|c|c|c|}
\hline \multirow[t]{2}{*}{ Mezcla } & \multicolumn{2}{|c|}{$\begin{array}{c}\text { Arena } \\
\text { con gravas }\end{array}$} & \multicolumn{2}{|c|}{$\begin{array}{c}\text { Arenas } \\
\text { gruesa y fina }\end{array}$} & \multicolumn{2}{|c|}{ Arcilla } & \multicolumn{2}{|c|}{$\begin{array}{l}\text { Cal Aérea } \\
\text { Hidratada }\end{array}$} & \multicolumn{2}{|c|}{$\begin{array}{l}\text { Cemento de } \\
\text { Albañilería }\end{array}$} & \multicolumn{2}{|c|}{$\begin{array}{l}\text { Cemento } \\
\text { Porthand }\end{array}$} \\
\hline & Cant & $\%$ & Cant & $\%$ & Cant & $\%$ & Cant & $\%$ & Cant & $\%$ & Cant & $\%$ \\
\hline Mezcla 1 & 12 & $60 \%$ & - & $0 \%$ & 4 & $20 \%$ & 2 & $10 \%$ & - & $0 \%$ & 2 & $10 \%$ \\
\hline Mezcla 2 & 8 & $40 \%$ & 4 & $20 \%$ & 4 & $20 \%$ & 2 & $10 \%$ & - & $0 \%$ & 2 & $10 \%$ \\
\hline Mezcla 3 & 8 & $40 \%$ & 4 & $20 \%$ & 4 & $20 \%$ & - & $0 \%$ & 2 & $10 \%$ & 2 & $10 \%$ \\
\hline Mezcla 4 & - & $0 \%$ & 12 & $60 \%$ & 4 & $20 \%$ & 2 & $10 \%$ & - & 0 & 2 & $10 \%$ \\
\hline Mezcla 5 & - & $0 \%$ & 12 & $60 \%$ & 4 & $20 \%$ & - & $0 \%$ & 3 & $15 \%$ & 1 & $5 \%$ \\
\hline Mezcla 6 & 8 & $60 \%$ & 4 & $0 \%$ & 3 & $15 \%$ & 2 & $0 \%$ & - & $10 \%$ & 2 & $10 \%$ \\
\hline
\end{tabular}




\section{Proceso de producción del bloque}

\section{Tamizado y pulverizado}

El suelo seleccionado es pasado por tamices de malla para eliminar los cantos rodados y las gravas (Fig.4). La arcilla es pulverizada en seco, mediante un proceso de molido y mezclada con el suelo. (Fig. 5)

Figura 4. Suelo y los tamices utilizados

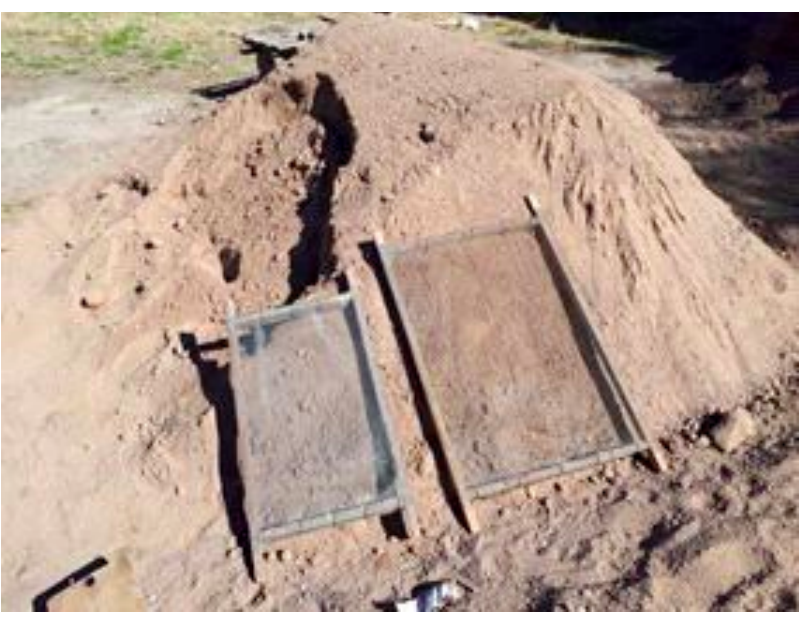

Fuente: Fotografía del autor.

\section{Mezclado e hidratado}

El suelo, la arcilla y el estabilizante son mezclados en seco en forma manual hasta lograr un color homogéneo. El hidratado se realiza rociando agua con una regadera de jardín, hasta alcanzar el contenido óptimo de humedad con el cual se obtiene la máxima densidad al compactar (Fig. 6)

\section{Moldeado}

Una vez hidratada, la mezcla es colocada en la prensa y compactada por el sistema de palancas (Figura 7). La extracción del bloque se realiza de igual manera mediante el sistema de palancas. (Fig. 8)
Figura 5. Pulverización de la arcilla

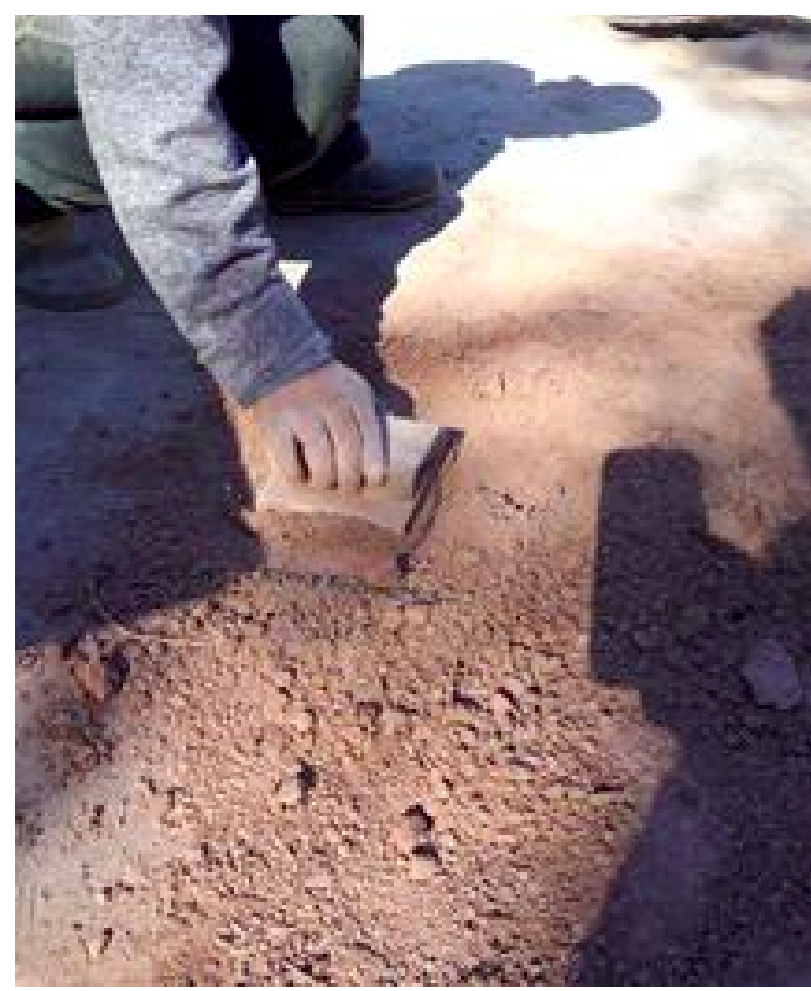

Fuente:" Fotografía del autor.

Figura 6. Mezclado del suelo y estabilizantes

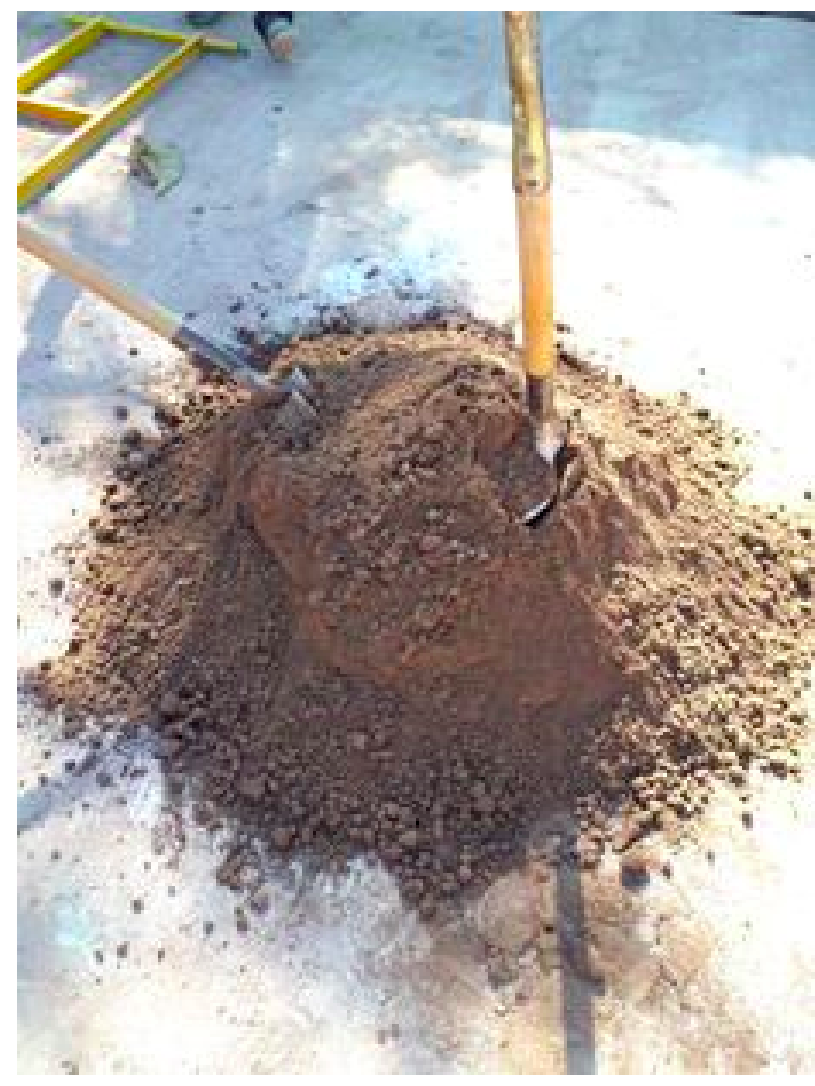

Fuente: Fotografía del autor. 
Figura 7. Llenado manual de la caja de la prensa

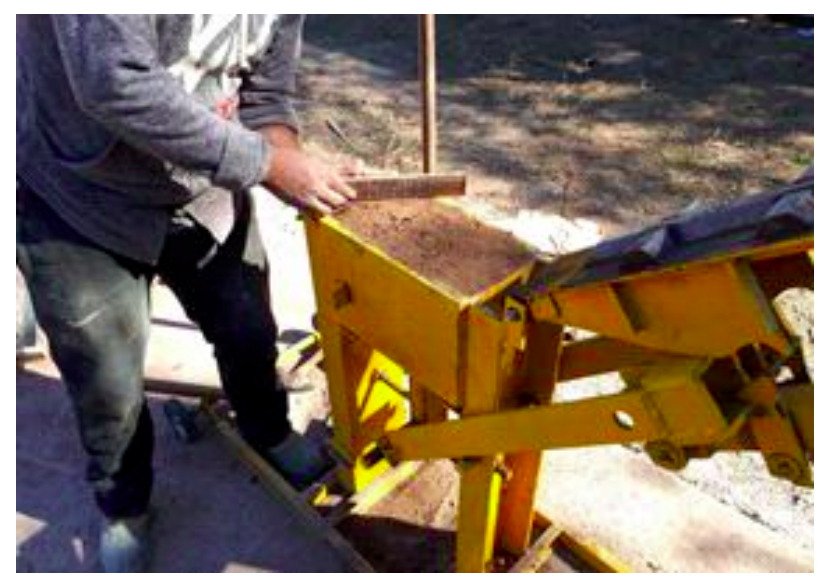

Fuente: Fotografía del autor

Figura 8. Compactación por medio del accionamiento del sistema de palancas

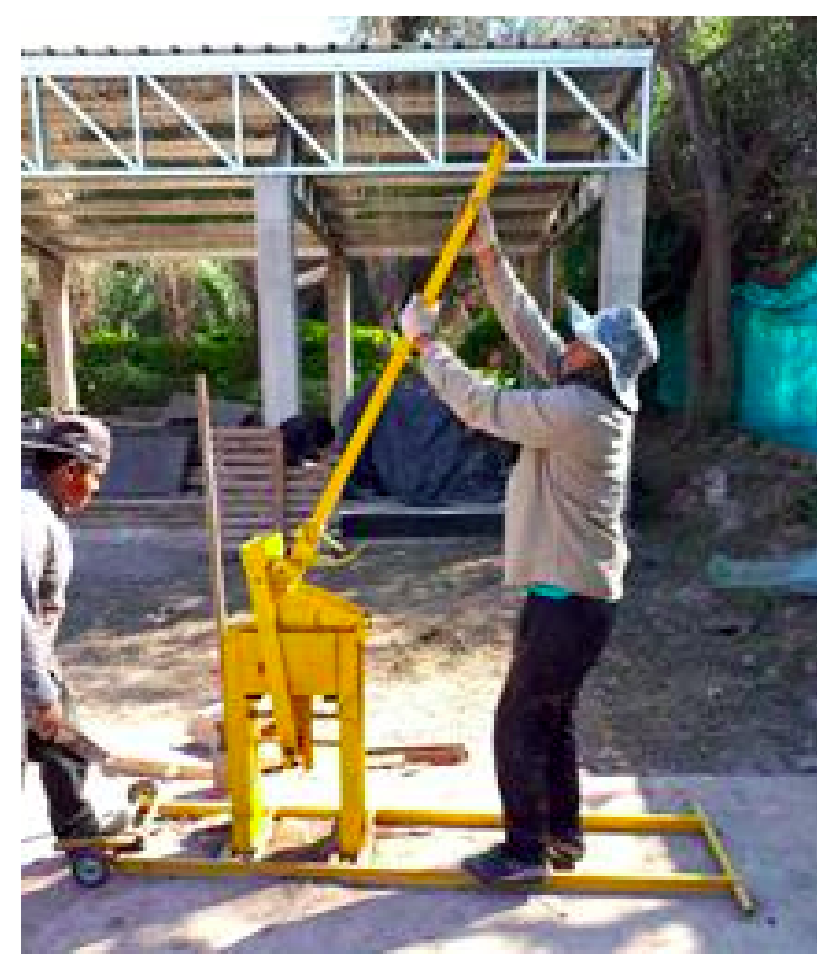

Fuente: Fotografía del autor.

\section{Curado}

EL proceso de curado del BSC demora aproximadamente una semana, dependido de las condiciones climáticas del lugar (humedad y temperatura), durante la cual los bloques son regados diariamente y mantenidos cubiertos bajo un plástico, retardando así su proceso de deshidratación y mejorar su fraguado. (Fig. 9)

Figura 9. Hidratación de los bloques durante el proceso de curado

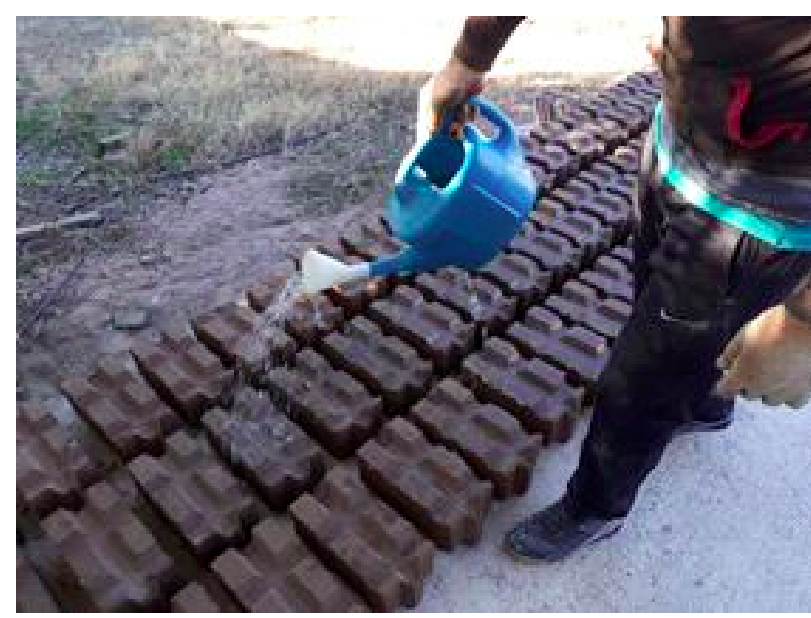

Fuente: Fotografía del autor

\section{Acopio}

Antes de ser utilizados los bloques son almacenados durante 20 días sobre "pallets" de madera que los protegen de la humedad natural del suelo y facilitar su traslado. (Fig. 10)

\section{Figura 10. Acopio del BSC sobre pallets}

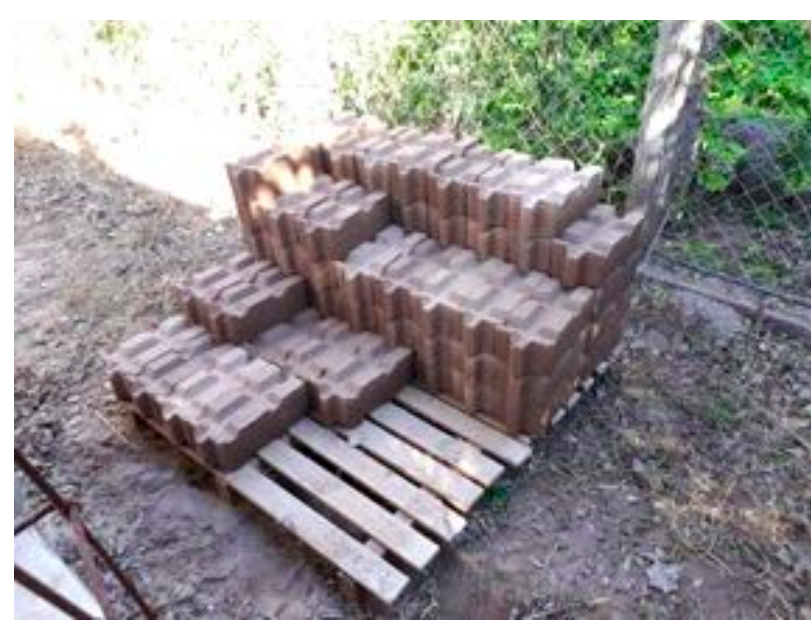

Fuente: Fotografía del autor. 


\section{Ejecución de Mampostería}

Para la ejecución de las mamposterías con BSC inter trabados fueron ensayados distintos tipos de morteros de asiento. Debido al poco espesor de las juntas y el tipo de encastre que no permite que la mezcla rebase por estas, los morteros a base de arenas a la cal o cementicos, no resultaron adecuados. Por su fluidez y maleabilidad el pegamento para pisos cerámicos resultó ser el más apropiado para el asiento de los bloques.

La primera hilada fue colocada sobre una capa de hormigón de $3 \mathrm{cms}$ de espesor tratada con dos manos de pintura asfáltica ${ }^{8}$, aislando así la mampostería de la humedad natural del terreno (Fig. 11)

Para asegurar la adherencia entre la mampostería y la estructura, en las juntas horizontales de los bloques cada tres hiladas fueron colocadas barras de hierros ancladas el interior de hormigón de las columnas. (Fig. 12)

\section{Costos de producción}

La prensa manual sin la tolva de carga, operada por dos personas produce 90 bloques diarios en una jornada de 8 hs; luego de ser instada la tolva la producción aumento hasta 140 bloques diarios, mejorando así su rendimiento en un $55 \%$.

En base al precio de los materiales utilizados y de los jornales de los operarios involucrados en el proceso, el costo total de producción del bloque es de $\$ 27,12$ (Tabla 2)

De las 8 hs de la jornada diaria, 4.5 hs. son necesarias para preparar el material: tamizar el suelo, moler la greda y mezclarlo con los estabilizantes y 3.5 hs para el moldeado del bloque, siendo la distribución de costos de producción la siguiente (Fig. 13)

\section{Figura 11. Detalle de la capa aisladora}

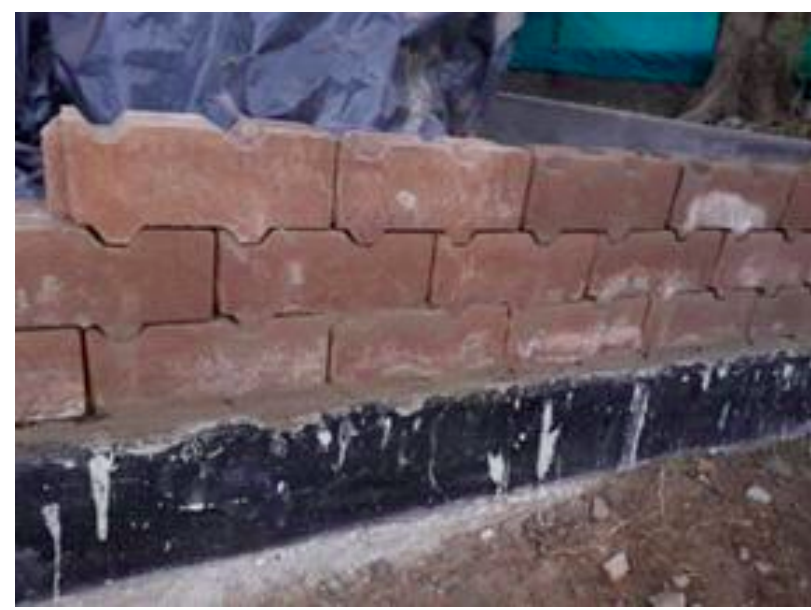

Fuente: Fotografía del autor.

Figura 12. Encuentro de una mampostería de BSC con la estructura de $\mathrm{H}^{\circ} \mathrm{A}$

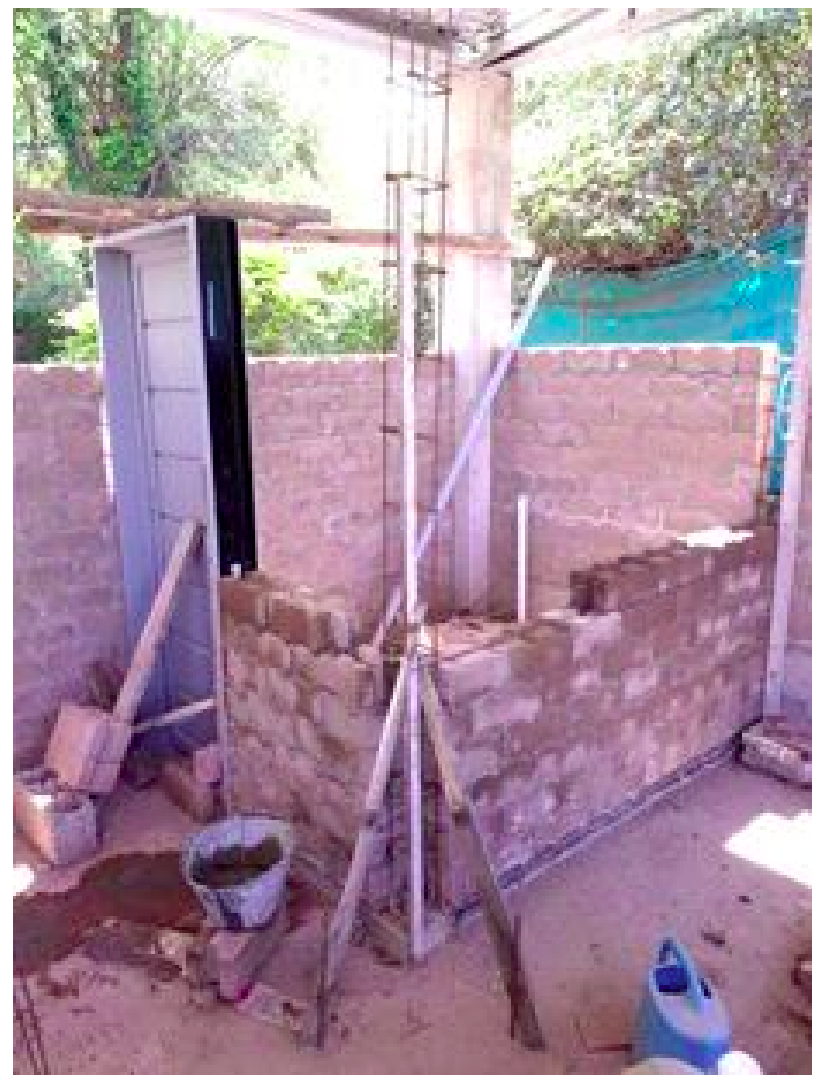

Fuente: Fotografía del autor.

\footnotetext{
${ }^{8}$ Se definen como pinturas asfálticas los productos fluidos constituidos por una base bituminosa, utilizados para la aplicación sobre materiales porosos con el fin de dotarlos de una película protectora resistente al paso de la humedad.
} 
Tabla 2. Análisis de costos de BSC realizado con prensa manual con prensa manual con tolva cargadora, Agosto de 2019, expresado en pesos argentinos

\begin{tabular}{|l|c|r|c|c|}
\hline \multicolumn{1}{|c|}{ ítems } & Unidad & Cantidad & $\begin{array}{c}\text { Precio } \\
\text { Unidad }\end{array}$ & Subtotal \\
\hline Suelos de río & M3 & 0.0350 & $\$ 150,00$ & $\$ 5,25$ \\
Arcilla & M3 & 0.0011 & $\$ 1.000,00$ & $\$ 1,11$ \\
Cemento de Albañilería & Bolsa & 0.0300 & $\$ 270,00$ & $\$ 8,10$ \\
Cemento Portland & Bolsa & 0.008 & $\$ 320,00$ & $\$ 2,67$ \\
Mano de obra preparado & Hora & 0.03214 & $\$ 175,00$ & $\$ 5.62$ \\
Mano de obra moldeado & Hora & 0.0250 & $\$ 175,00$ & $\$ 4.37$ \\
\hline \multicolumn{7}{|c|}{} \\
\hline \multicolumn{7}{|c|}{ TOTAL } & $\$ 27.12$ \\
\hline
\end{tabular}

Fuente: Elaboración propia

Figura 13. Distribución de costos de producción de BSC con prensa manual

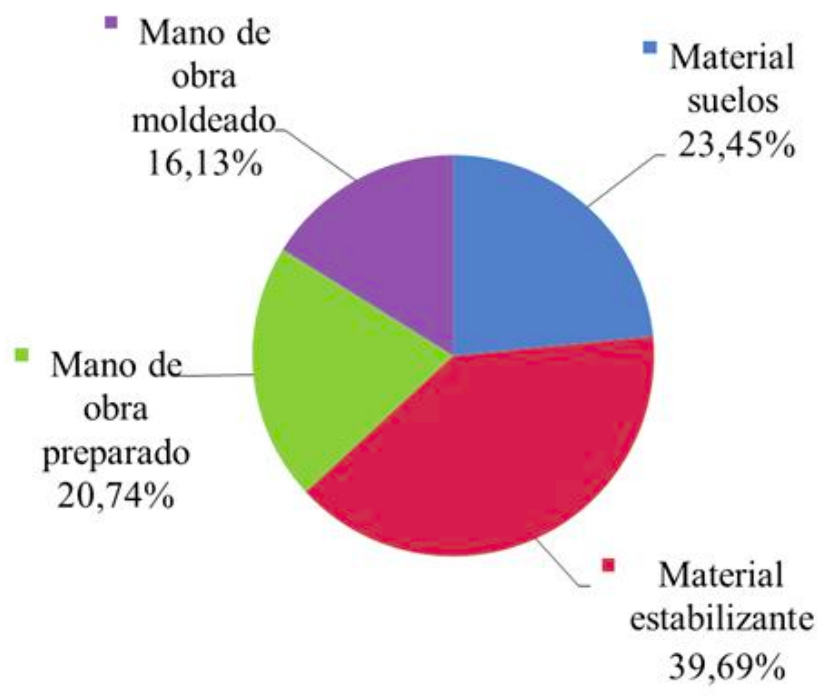

Fuente: Elaboración propia.

\section{RESULTADOS}

Durante la ejecución de las mamposterías fueron tomadas mediciones del rendimiento de los materiales y el tiempo empleado por los operarios para realizar esta tarea. (Tabla 3)

Tabla 3. Análisis de costos de BSC por M2, Agosto de 2019, expresado en pesos argentinos

\begin{tabular}{|l|c|c|r|r|}
\hline \multicolumn{1}{|c|}{ ítems } & Unidad & Cantidad & $\begin{array}{r}\text { Precio } \\
\text { Unidad }\end{array}$ & Subtotal \\
\hline Bloque de Suelo Cemento & Unid & 25,00 & $\$ 27,12$ & $\$ 678,00$ \\
\hline Pegamento para cerámico & Bolsa & 0,197 & $\$ 255,00$ & $\$ 50,25$ \\
Mano de Obra oficial & hs & 1,98 & $\$ 138,81$ & $\$ 274,92$ \\
Mano de obra ayudante & hs & 1,56 & $\$ 117,49$ & $\$ 183,28$ \\
\hline \multicolumn{5}{r}{} \\
\end{tabular}

Fuente: Elaboración propia

Al comparar la mampostería de BSC con otras mamposterías realizadas con materiales industrializados, podemos observar que su costo no es mucho mayor que el de las mamposterías de bloques de hormigón y de ladrillo cerámico huecos, las cuales no tienen la aislación térmica que tiene esta. Al compararla con la mampostería de ladrillos macizos calcinados, el mampuesto de BSC es de menor precio, ya que compensa sus mayor costo de producción con un menor costo en la ejecución de la misma por su sistema de encastre. (Tabla 4)

Tabla 4. Cuadro comparativo de costos de distintos tipos de mampostería, Agosto de 2019, expresado en pesos argentinos

\begin{tabular}{|l|c|c|c|c|}
\hline \multicolumn{1}{|c|}{ Mampostería } & Und. & Material & $\begin{array}{c}\text { Mano } \\
\text { de Obra }\end{array}$ & Total \\
\hline De bloques de Suelo Cemento & M2 & $\$ 728,25$ & $\$ 458,20$ & $\$ 1186,45$ \\
De bloques de hormigón & M2 & $\$ 493,65$ & $\$ 618,90$ & $\$ 1112,55$ \\
De ladrillo cerámico hueco & M2 & $\$ 586,33$ & $\$ 521,28$ & $\$ 1107,61$ \\
De ladrillos macizo de horno & M2 & $\$ 499,22$ & $\$ 908,40$ & $\$ 1407,62$ \\
\hline
\end{tabular}

Fuente: Revista Vivienda $\mathrm{N}^{\circ} 685$ 


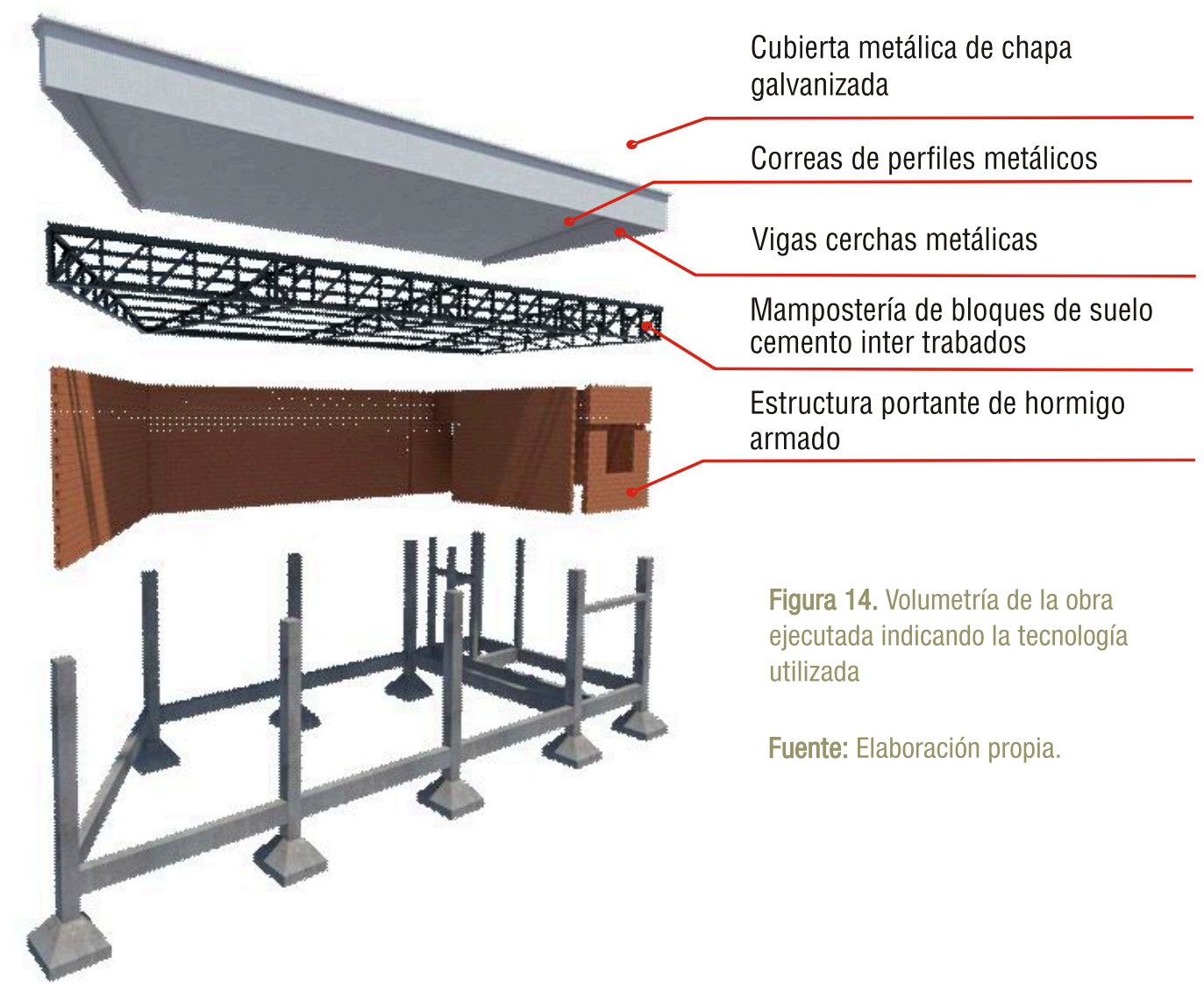

\section{DISCUSIÓN}

La producción de BSC mediante una prensa de accionamiento manual es muy inferior a la que los fabricantes de estas máquinas indican poder lograr, generando falsas expectativas entre quienes optan por esta tecnológica.

Hay muchas publicaciones en las que se afirma que cualquier persona puede producir estos bloques, inclusive hay quienes ven en esta tecnología una posibilidad económica, cuando en realidad para lograr un producto de calidad es necesario poseer sólidos conocimientos sobre mecánica de suelos y su estabilización.

Esta realidad se comprueba en la ciudad de La Rioja en donde en la actualidad existen casi diez prensas para bloques de suelo adquiridas para realizar emprendimientos comerciales que no han prosperado, por falta de conocimientos del material o por expectativas de producción que no se acerca a la realidad.

\section{CONCLUSIONES}

Los suelos aptos para producir BSC son aquellos que poseen una distribución granulométrica uniforme estando presentes todas las granulometrías, de modo que las partículas más finas ocupan los espacios vacantes entre las partículas más grandes, alcanzado asi la máxima densidad al ser compactados.

No es favorable la presencia de particulares mayores a $2 \mathrm{~mm}$. (gravas) en los suelos utilizados para producir BSC.

Elaborar BSC con suelos transportados desde una cantera encarece su costo en un $23 \%$, por tal motivo es importante detectar una fuente de suelo apto lo más cercana posible al lugar de producción.

Siendo el costo de preparación del material una parte importante del costo de producción del bloque (21\%), resulta 
conveniente optimizar este proceso incorporando una zaranda automática o un sistema de molienda de la arcilla.

La estabilización de BSC mediante cemento de albañilería es una buena alternativa al uso de cal, la cual es un producto poco normalizado que puede tener problemas de calidad y al uso de cemento portland, de mayores costos.

Si bien el inter trabado de doble machihembrado mejora la resistencia a la tracción diagonal en las mamposterías de BSC, este sistema de encastre no permite biselar las aristas del bloque con el consiguiente problema de desgranamiento y defectos en las terminaciones.

La mampostería de BSC realizadas con bloques producidos en una prensa manual tiene costos competitivos respecto a otras mamposterías realizadas con materiales industrializados de menores prestaciones térmicas.

Es ineludible realizar ensayos al suelo utilizado para establecer científicamente sus características: granulometría, límite líquido, límite plástico, presencia de sales solubles y sulfatos, utilizando para ello las normas de ensayo para suelos de la Dirección Nacional de Vialidad.

Los bloques producidos deberán ser ensayos a la compresión simple, a la erosión acelerada por goteo y a la absorción de agua por capilaridad. No habiendo normas de ensayo para estos bloques en Argentina, se propone utilizar la norma europea AENOR UNE 41410.

Una sección de mampostería de BSC producida deberá ser ensayada a la compresión simple y a la compresión diagonal, según lo establece la norma CIRSOC 103 del IMPRES.

\section{REFERENCIAS BIBLIOGRÁFICAS}

IMCyC Instituto Mexicano del Cemento y el Concreto (2008) Documento Suelo Cemento, parte 1.

http://www.imcyc.com/ct2008/mar08/ingenie ria.htm

Neves C.; Faria Borges O; Rotondaro R.; Cevallos P.; Hoffmann M.; (2011) Selección de Suelos y Métodos de Control en la Construcción con Tierra, prácticas de campo. Bauru: FEBUNESP/PROTERRA. Disponible en $<$ http://www.redproterra.org>

DNV Dirección Nacional de Vialidad de la República Argentina (1989) Normas de Ensayos

AENOR, Asociación Española de Normalización y Certificación (2008) UNE 41410, Bloques de tierra comprimida para muros y tabiques, definiciones, especificaciones y métodos de ensayos. Madrid, España.

INPRES, Instituto Nacional de Previsión Sísmica, (2018) Reglamento Argentino para la Construcciones Simoresistentes, parte III, Construcciones de Mamposterías.

\section{AGRADECIMIENTOS}

El autor agradece la colaboración de los arquitectos Luis Alfredo Orecchia y Verónica Mariana Vargas por las correcciones estilística del texto; Joaquín Garcé por la traducción al inglés del resumen y Cristian Allegretti por la gráfica del proyecto.

\section{CORRESPONDENCIA}

Eduardo Enrique Brizuela

Profesional independiente, Argentina.

enribrizu@yahoo.com.ar 